\title{
Mixed aliphatic and aromatic composition of evaporating very small grains in NGC 7023 revealed by the $3.4 / 3.3 \mu \mathrm{m}$ ratio
}

\author{
P. Pilleri ${ }^{1,2}$, C. Joblin ${ }^{1,2}$, F. Boulanger ${ }^{3}$, and T. Onaka ${ }^{4}$ \\ ${ }^{1}$ Université de Toulouse, UPS-OMP, IRAP, BP 44346, 31028 Toulouse, France \\ e-mail: paolo.pilleri@irap.omp.eu \\ 2 CNRS, IRAP, 9 Av. colonel Roche, BP 44346, 31028 Toulouse Cedex 4, France \\ 3 Institut d'Astrophysique Spatiale, 91405 Orsay, France \\ ${ }^{4}$ Department of Astronomy, Graduate School of Science, University of Tokyo, 113-0033 Tokyo, Japan
}

Received 27 December 2014 / Accepted 17 February 2015

\section{ABSTRACT}

\begin{abstract}
Context. A chemical scenario was proposed for photon-dominated regions (PDRs) according to which UV photons from nearby stars lead to the evaporation of very small grains (VSGs) and the production of gas-phase polycyclic aromatic hydrocarbons (PAHs). Aims. Our goal is to achieve better insight into the composition and evolution of evaporating very small grains (eVSGs) and PAHs through analyzing the infrared (IR) aliphatic and aromatic emission bands.

Methods. We combined spectro-imagery in the near- and mid-IR to study the spatial evolution of the emission bands in the prototypical PDR NGC 7023. We used near-IR spectra obtained with the IRC instrument onboard AKARI to trace the evolution of the $3.3 \mu \mathrm{m}$ and $3.4 \mu \mathrm{m}$ bands, which are associated with aromatic and aliphatic $\mathrm{C}-\mathrm{H}$ bonds on PAHs. The spectral fitting involved an additional broad feature centered at $3.45 \mu \mathrm{m}$ that is often referred to as the plateau. Mid-IR observations obtained with the IRS instrument onboard the Spitzer Space Telescope were used to distinguish the signatures of eVSGs and neutral and cationic PAHs. We correlated the spatial evolution of all these bands with the intensity of the UV field given in units of the Habing field $G_{0}$ to explore how their carriers are processed.

Results. The intensity of the $3.45 \mu \mathrm{m}$ plateau shows an excellent correlation with that of the $3.3 \mu \mathrm{m}$ aromatic band (correlation coefficient $R=0.95)$ and a relatively poor correlation with the aliphatic $3.4 \mu \mathrm{m}$ band $(R=0.77)$. This indicates that the $3.45 \mu \mathrm{m}$ feature is dominated by the emission from aromatic bonds. We show that the ratio of the $3.4 \mu \mathrm{m}$ and $3.3 \mu \mathrm{m}$ band intensity $\left(I_{3.4} / I_{3.3}\right)$ decreases by a factor of 4 at the PDR interface from the more UV-shielded layers $\left(G_{0} \sim 150, I_{3.4} / I_{3.3}=0.13\right)$ to the more exposed layers $\left(G_{0}>1 \times 10^{4}, I_{3.4} / I_{3.3}=0.03\right)$. The intensity of the $3.3 \mu \mathrm{m}$ band relative to the total neutral PAH intensity shows an overall increase with $G_{0}$, associated with an increase of both the hardness of the UV field and the $\mathrm{H}$ abundance. In contrast, the intensity of the $3.4 \mu \mathrm{m}$ band relative to the total neutral PAH intensity decreases with $G_{0}$, showing that their carriers are actively destroyed by UV irradiation and are not efficiently regenerated. The transition region between the aliphatic and aromatic material is found to correspond spatially with the transition zone between neutral PAHs and eVSGs.

Conclusions. We conclude that the photo-processing of eVSGs leads to the production of PAHs with attached aliphatic sidegroups that are revealed by the $3.4 \mu \mathrm{m}$ emission band. Our analysis provides evidence for the presence of very small grains of mixed aromatic and aliphatic composition in PDRs.
\end{abstract}

Key words. photon-dominated region (PDR) - ISM: individual objects: NGC 7023 - ISM: molecules

\section{Introduction}

A significant fraction of interstellar carbon (up to $20 \%$, Joblin et al. 1992; Tielens 2005) is tied up in the carriers of the aromatic infrared bands (AIBs). The most intense AIBs are observed at $3.3,6.2,7.7,8.6,11.3$, and $12.7 \mu \mathrm{m}$ and are generally attributed to stochastically heated polycyclic aromatic hydrocarbons (PAHs, Léger \& Puget 1984; Allamandola et al. 1985). This set of emission bands shows systematic variations in the relative intensity and central wavelength of the bands (Peeters et al. 2002), reflecting variations in the local physical conditions and a chemical evolution of their carriers. From the analysis of spectro-imagery data on several Galactic photon-dominated regions (PDRs), the variations of the mid-IR $(5.5-15 \mu \mathrm{m})$ bands were attributed to the emission of three different populations: $\mathrm{PAH}$ cations and neutrals $\left(\mathrm{PAH}^{+}\right.$and $\left.\mathrm{PAH}^{0}\right)$ and evaporating very small grains (eVSGs, Rapacioli et al. 2005; Berné et al. 2007; Pilleri et al. 2012). A chemical scenario has been proposed according to which eVSGs evaporate under UV irradiation, producing free-flying PAHs. Candidates such as PAH clusters
(Rapacioli et al. 2006; Montillaud \& Joblin 2014) or Fe-PAH complexes (Simon \& Joblin 2009) have been proposed as models for these eVSGs. Jones (2012) recently proposed a model for the evolution of carbonaceous dust in the interstellar medium in which the smallest particles are aromatized, whereas the largest ones remain H-rich and predominantly aliphatic. Such a scenario would reconcile the observation of both the aromatic emission bands in UV-irradiated regions and the $3.4 \mu \mathrm{m}$ $\mathrm{CH}$ aliphatic absorption feature as a main feature of interstellar dust in galaxies (Dartois et al. 2004). To develop this evolutionary scenario further, we report here a study of the $3.4 \mu \mathrm{m}$ emission band, which is observed as a minor band at the side of the main AIB at $3.3 \mu \mathrm{m}$. Characteristic of aliphatic $\mathrm{CH}$ bonds, this band has been attributed to methyl sidegroups attached to PAHs (Jourdain de Muizon et al. 1990) or to superhydrogenated PAHs (Bernstein et al. 1996). The observed 3.4/3.3 $\mu \mathrm{m}$ intensity ratio $\left(I_{3.4} / I_{3.3}\right)$ is found to decrease for an increasing intensity of the UV field (Geballe et al. 1989; Joblin et al. 1996; Sloan et al. 1997; Mori et al. 2014), reflecting the photo-destruction of the more fragile bonds linked to the $3.4 \mu \mathrm{m}$ band. 

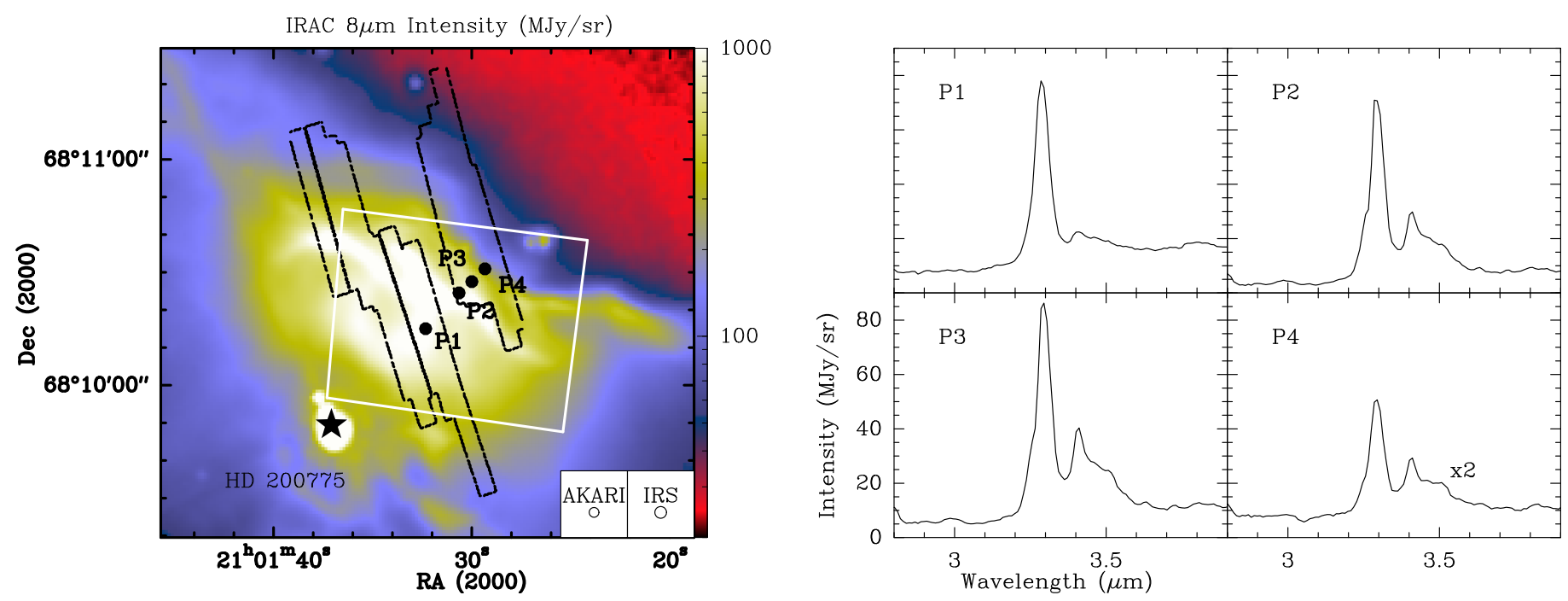

Fig. 1. In color scale, the Spitzer IRAC $8 \mu \mathrm{m}$ image of the NGC 7023 NW PDR (Werner et al. 2004). The dark lines demarcate the region in which AKARI-IRC data were obtained, the white rectangle represents the Spitzer-IRS field of view. The star represents the position of HD 200775. The insets represent the AKARI and IRS beam sizes. AKARI spectra between 2.8 and $3.9 \mu \mathrm{m}$ are shown to the right. They correspond to the positions P1-P4 that are marked as black dots on the map.

In this paper we present $2 \mathrm{D}$ spectroscopy of the PDR associated with the reflection nebula NGC $7023 \mathrm{NW}$ obtained with the AKARI and Spitzer space telescopes. The combination of these datasets allows us to study the spatial variations of the 3.3 and $3.4 \mu \mathrm{m}$ emissions due to aromatic and aliphatic bonds with those of the $\mathrm{PAH}^{0}$ and eVSG emissions. In Sect. 2 we present the observations and the data reduction. In Sect. 3 we describe the data analysis tools and present the observational results. We discuss these results in Sect. 4. Finally, the conclusions are presented in Sect. 5 .

\section{Observations}

\subsection{NGC 7023 NW}

NGC 7023 is a reflection nebula in the Cepheus constellation illuminated by the B2-5Ve binary star HD 200775 (Alecian et al. 2008). The distance of HD 200775 to the Sun measured by HIPPARCOS is $430_{-90}^{+160} \mathrm{pc}$, but a recent analysis revised the distance to $320 \pm 51 \mathrm{pc}$ (Benisty et al. 2013). The nebula has been shaped by the star formation process, which led to the formation of a cavity (Fuente et al. 1993). The interaction of the UV photons with the walls of the parent molecular cloud has produced several PDRs to the northwest, south, and east of the star. The brightest PDR is found about $45^{\prime \prime} \mathrm{NW}$ from the binary star (hereafter the NW PDR, or NGC 7023 NW). Pilleri et al. (2012) estimated the intensity of the radiation field at the PDR front to be $G_{0} \sim 2600$ in units of the Habing field (Habing 1968). Previous observations of different gas lines have shown that this region hosts structures at different gas densities: $n_{\mathrm{H}} \sim 100 \mathrm{~cm}^{-3}$ in the cavity (Berné \& Tielens 2012), $10^{5}-10^{6} \mathrm{~cm}^{-3}$ in the filaments that are observed in the mm (Fuente et al. 1996) and near-IR (Lemaire et al. 1996; Martini et al. 1997), and $\sim 10^{4} \mathrm{~cm}^{-3}$ in the molecular cloud (Gerin et al. 1998).

\subsection{Near- and mid-IR observations with Spitzer and AKARI}

The infrared camera (IRC, Onaka et al. 2007) onboard the AKARI space telescope (Murakami et al. 2007) was used to obtain near-IR $(2.5-5 \mu \mathrm{m})$ spectro-imagery of NGC $7023 \mathrm{NW}$. The observations were performed as part of the open-time program NESID of the AKARI post-helium phase between October 2008 and January 2010. They were carried out using the grism spectroscopy mode with the narrow Nh slit, which provides a spectral resolution of about $0.02 \mu \mathrm{m}$ and a spatial resolution of $\sim 3^{\prime \prime}$ (Onaka et al. 2007). The slit position was slightly shifted for the different runs to make dithering observations. In each pointed observation, eight to nine exposure cycles in spectroscopy were obtained together with one exposure in imaging in the $\mathrm{N} 3(3.2 \mu \mathrm{m})$ band, which gives the accurate position information. The on-source integration time for each observation is typically $360 \mathrm{~s}$. The data were processed with the latest version of the spectroscopic toolkit (version 20110301) for the phase 3 observation ${ }^{1}$. The NGC $7023 \mathrm{NW}$ observations consisted of 20 stripes of length $\sim 50^{\prime \prime}$, each with a slightly different position and orientation. The stripes were then combined in a mosaic using the software montage ${ }^{2}$. The field of view of the final mosaic results from the overlap of the slit coverages of all the stripes and is displayed in Fig. 1. The near-IR features vary smoothly between nearby positions but are significantly different with distance to the star, as shown by the four representative spectra in Fig. 1 that correspond to offsets of P1 [-29", 24"];

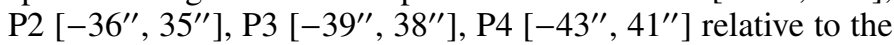
position of HD 200775.

We compare this dataset with the Spitzer-IRS observations of NGC 7023, which provides spectral imagery in the wavelength range [5.5-14] $\mu \mathrm{m}$ and covers the whole filamentary region of the NW PDR with a spatial resolution of 3.6" (see Fig. 1). This dataset has been described in detail in previous studies, for example, Werner et al. (2004), Berné et al. (2007), and Pilleri et al. (2012).

\section{Spectral analysis and spatial distributions}

\subsection{Near-infrared}

The first column of Fig. 2 shows the AKARI-IRC spectra observed at the positions P1-P4. The spectra are dominated by the

\footnotetext{
http://www.ir.isas.jaxa.jp/ASTRO-F/Observation/

DataReduction/IRC/

2 http://montage.ipac.caltech.edu
} 

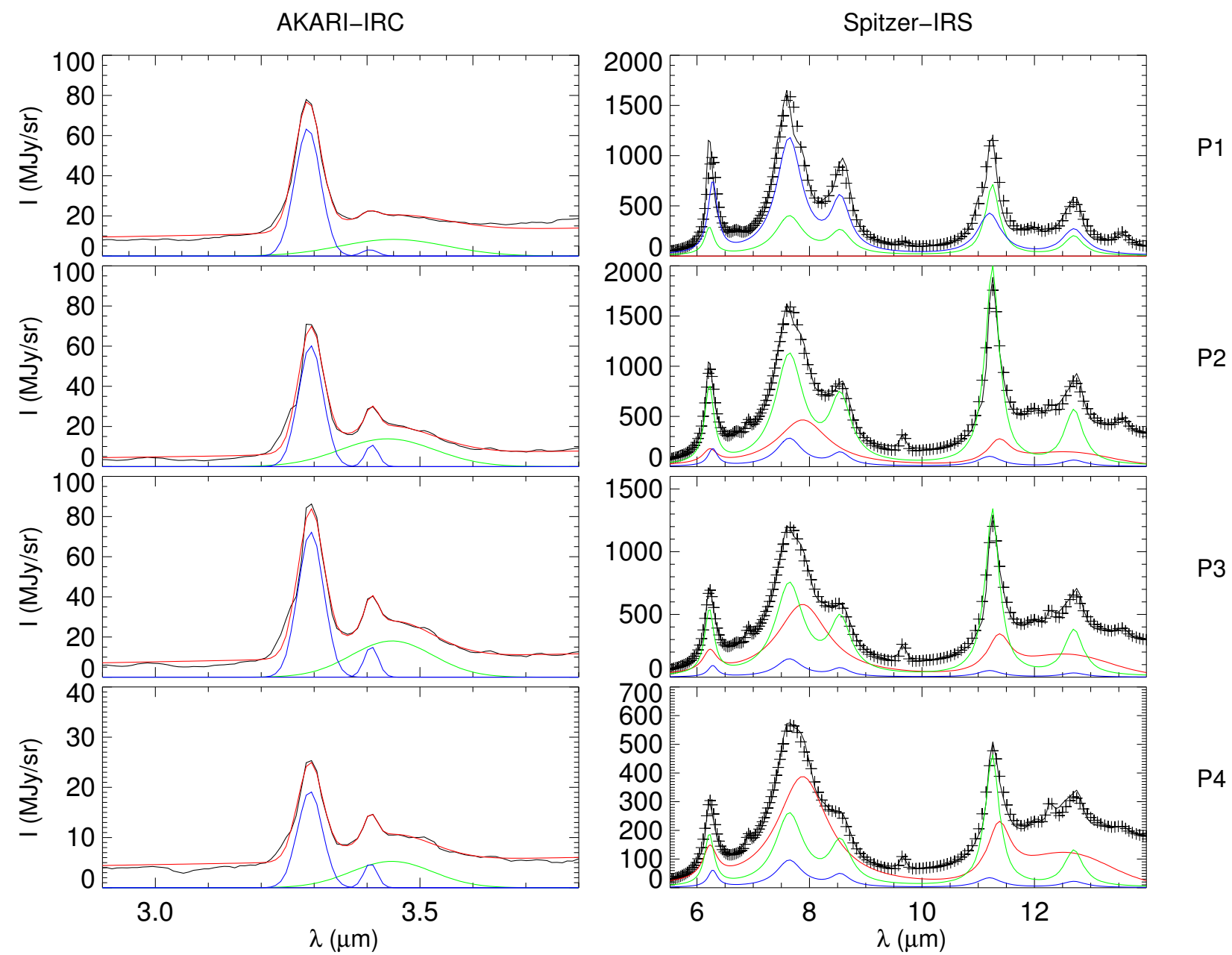

Fig. 2. Summary of the observations and spectral analysis for positions $\mathrm{P} 1$ to $\mathrm{P} 4$ (top to bottom row). First column: the observed spectrum (black) and its fit (red) for the AKARI observations. The fit of the 3.29 and $3.40 \mu \mathrm{m}$ features are displayed in blue, and the $3.45 \mu \mathrm{m}$ plateau is shown in green. Second column: observed Spitzer-IRS spectrum (crosses), its fit (black) and the decomposition obtained with PAHTAT (red: eVSG, green: $\mathrm{PAH}^{0}$, blue: $\left.\mathrm{PAH}^{+}\right)$.

two emission bands that peak at 3.29 and $3.40 \mu \mathrm{m}$, in addition to an underlying plateau. To fit these spectra we used two narrow Gaussians for the 3.29 and $3.40 \mu \mathrm{m}$ features and a broader Gaussian centered at $3.45 \mu \mathrm{m}$ to account for the plateau. We let the central wavelength of the Gaussians vary by $0.01 \mu \mathrm{m}$ to account for small shifts and treated the band widths and intensities as free parameters. We simultaneously fit the observed spectrum using a linear combination of these features and a linear continuum. The resulting full widths at half maximum of the bands were found to be $\sim 0.03 \mu \mathrm{m}$ for the 3.29 and $3.40 \mu \mathrm{m}$ bands and $\sim 0.1 \mu \mathrm{m}$ for the $3.45 \mu \mathrm{m}$ plateau. As shown in Fig. 2, this yields very good fits of the spectra, even in cases with a strong plateau and faint superposed $3.40 \mu \mathrm{m}$ band.

The $3.29 \mu \mathrm{m}$ band is commonly attributed to the $\mathrm{C}-\mathrm{H}$ inplane stretching mode in PAHs, whereas the $3.40 \mu \mathrm{m}$ band and the plateau are more difficult to attribute unequivocally. Steglich et al. (2013) concluded that it is difficult from a spectroscopic point of view to favor the attribution of the $3.4 \mu \mathrm{m}$ band either to methyl side groups attached to PAHs or to superhydrogenated PAHs. Still, Joblin et al. (1996) showed that the evolution of the $3.4 / 3.3 \mu \mathrm{m}$ band ratio with the UV field can be explained by methylated PAHs. The $3.45 \mu \mathrm{m}$ plateau is most likely the superposition of a number of faint features including other aliphatic modes, hot band emission of aromatic $\mathrm{CH}$ (Barker et al. 1987) and combination bands of $\mathrm{C}-\mathrm{C}$ and
$\mathrm{C}-\mathrm{H}$ aromatic modes (Allamandola et al. 1989). In Fig. 3 we show the pixel-to-pixel correlation of the integrated intensity of the $3.45 \mu \mathrm{m}$ plateau $\left(I_{\text {pla }}\right)$ with that of the $3.29 \mu \mathrm{m}\left(I_{3.3}\right)$ and $3.40 \mu \mathrm{m}\left(I_{3.4}\right)$ bands. $I_{\text {pla }}$ correlates better with $I_{3.3}$ (correlation coefficient $R=0.95)$ than with $I_{3.4}(R=0.77)$. This suggests that the plateau is dominated by the emission from aromatics (hot bands and combination bands). Since our goal is to study the behavior of the aromatic vs. aliphatic evolution, we exclude the plateau from the analysis in the following.

\subsection{Mid-infrared}

To extract the emission of the different AIB carriers, we used the results of the PAHTAT procedure (Pilleri et al. 2012). Figure 2 shows the mid-IR spectra and the corresponding fits for the four positions P1-P4. PAHTAT also allows estimating the value of $A_{\mathrm{V}}$ along the line of sight assuming that the AIB carriers and other dust populations are well mixed. From these $A_{\mathrm{V}}$ values, we reconstructed the deredenned mid-IR and near-IR spectra by using the extinction curve from Weingartner \& Draine (2001) for an $R_{\mathrm{V}}$ value of 5.6 (Witt et al. 2006). These unreddened spectra are used in the following analysis. The 3.3 and $3.4 \mu \mathrm{m}$ bands are sufficiently close in wavelength for their ratio is to be not significantly affected by the extinction correction. 

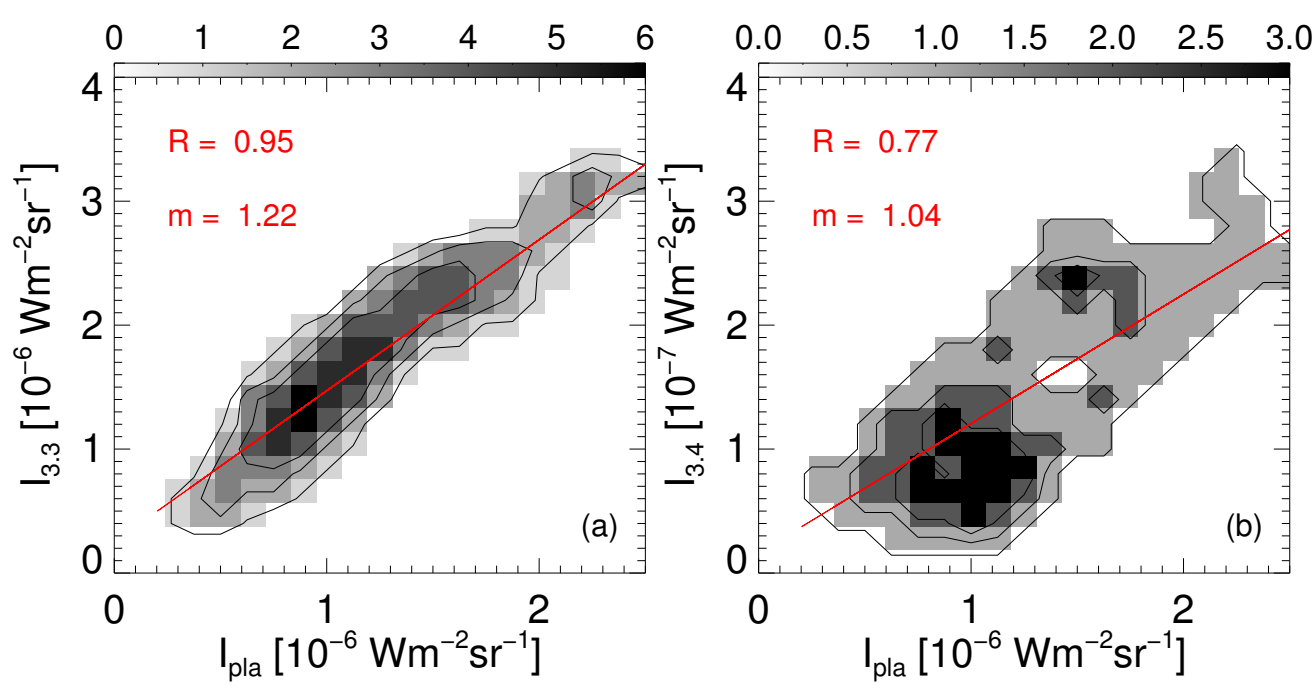

Fig. 3. Pixel-to-pixel correlations of the integrated intensity of the $3.3 \mu \mathrm{m}$ band $\left(I_{3.3}\right), 3.4 \mu \mathrm{m}$ band $\left(I_{3.4}\right)$, and the $3.45 \mu \mathrm{m}$ plateau $\left(I_{\text {pla }}\right)$. The correlation coefficient $R$ and the slope $m$ of the linear fit to the points are also given. $I_{\text {pla }}$ correlates better with the intensity of the aromatic $3.3 \mu \mathrm{m}$ band than with the aliphatic $3.4 \mu \mathrm{m}$ band. This indicates that the plateau is dominated by emission from aromatic bonds.

Table 1. Results of the fit of the mid- and near-IR observations.

\begin{tabular}{c|c|cccc}
\hline \hline & Unit & $\mathrm{P} 1$ & $\mathrm{P} 2$ & $\mathrm{P} 3$ & $\mathrm{P} 4$ \\
\hline$I_{\mathrm{eVSG}}$ & $10^{-5} \mathrm{~W} \mathrm{~m}^{-2} \mathrm{sr}^{-1}$ & 0.00 & 4.81 & 6.00 & 4.01 \\
$I_{\mathrm{PAH}}$ & $10^{-5} \mathrm{~W} \mathrm{~m}^{-2} \mathrm{sr}^{-1}$ & 4.04 & 11.4 & 7.60 & 2.63 \\
$I_{\mathrm{PAH}^{+}}$ & $10^{-5} \mathrm{~W} \mathrm{~m}^{-2} \mathrm{sr}^{-1}$ & 8.87 & 2.13 & 1.10 & 0.73 \\
$I_{\mathrm{AIB}}$ & $10^{-5} \mathrm{~W} \mathrm{~m}^{-2} \mathrm{sr}^{-1}$ & 12.9 & 18.3 & 14.7 & 7.36 \\
\hline$A_{\mathrm{V}}$ & $\mathrm{mag}$ & $<2$ & 15 & 19 & 23 \\
$f_{\mathrm{eVSG}}$ & - & 0.00 & 0.42 & 0.58 & 0.71 \\
$G_{0}$ & - & 7000 & 2600 & 200 & 150 \\
\hline$I_{3.3}$ & $10^{-7} \mathrm{~W} \mathrm{~m}^{-2} \mathrm{sr}^{-1}$ & 11.0 & 15.8 & 22.3 & 6.47 \\
$I_{3.4}$ & $10^{-7} \mathrm{~W} \mathrm{~m}^{-2} \mathrm{sr}^{-1}$ & 0.31 & 1.42 & 2.34 & 0.80 \\
$I_{\mathrm{pla}}$ & $10^{-7} \mathrm{~W} \mathrm{~m}^{-2} \mathrm{sr}^{-1}$ & 5.36 & 13.5 & 15.6 & 4.80 \\
$I_{3.4} / I_{3.3}$ & - & 0.028 & 0.090 & 0.11 & 0.13 \\
\hline
\end{tabular}

Table 1 shows the relevant results of the fit for the positions P1-P4, that is, the integrated intensity of the near-IR bands, of the $\mathrm{PAH}^{+}, \mathrm{PAH}^{0}$, eVSG, and of the total AIBs, as well as the fraction of carbon locked in eVSGs $\left(f_{\mathrm{eVSG}}\right)$. In Pilleri et al. (2012), we have shown that $f_{\text {evSG }}$ can be derived from every pixel and then used as a probe for $G_{0}$ in the region of the PDR with significant eVSG emission. For NGC $7023 \mathrm{NW}$, this region starts at a distance of $\sim 45^{\prime \prime}$ from HD 200775, corresponding to $G_{0}=2600$. In the more exposed layers of the PDR, we obtain an estimate of the UV field intensity by assuming geometrical dilution of the stellar radiation field, a stellar temperature of $T_{\text {eff }}=15000 \mathrm{~K}$ (Finkenzeller 1985), and a local extinction of $A_{\mathrm{V}}=1.5$ (Pilleri et al. 2012). The total map of $G_{0}$ we obtain is shown in Fig. 4.

\subsection{Spatial distributions}

Applied to the full IRS data cube, PAHTAT allows deriving the spatial distribution of the intensity of $\mathrm{PAH}^{+}, \mathrm{PAH}^{0}$, and eVSGs (top panel in Fig. 5). These results have been described in detail in Pilleri et al. (2012) and can be summarized as follows: $\mathrm{PAH}^{+}$dominate the mid-IR emission between the illuminating star and the PDR front; $\mathrm{PAH}^{0}$ are the most abundant species at the PDR front as traced by the emission of the $\mathrm{H}_{2}$ rotational lines; eVSGs dominate the emission slightly deeper inside the cloud; the transition between $\mathrm{PAH}^{0}$ and $\mathrm{eVSG}$ corresponds to

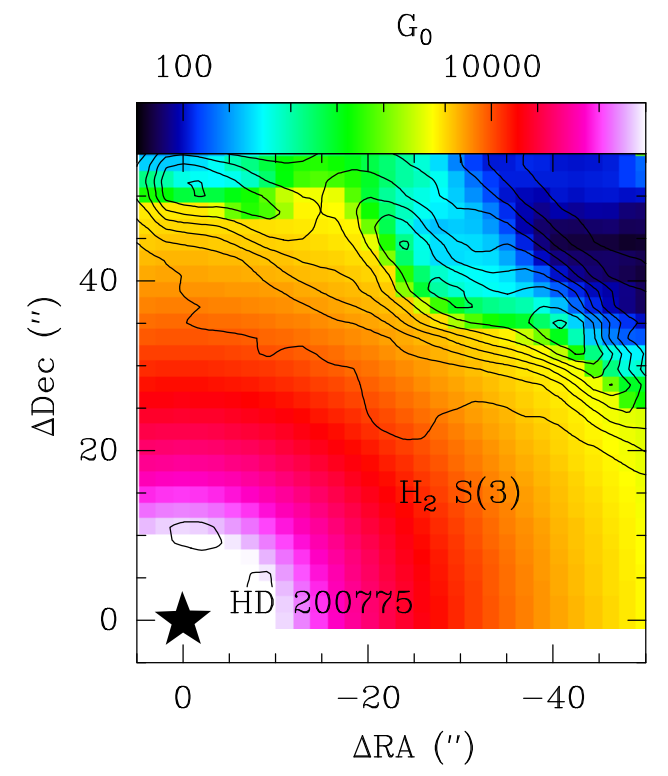

Fig. 4. Map of $G_{0}$ in NGC $7023 \mathrm{NW}$ (color scale). The $\mathrm{H}_{2} \mathrm{~S}(3)$ integrated intensity is shown for reference (linear steps of $1.7 \times$ $\left.10^{-7} \mathrm{~W} \mathrm{~m}^{-2} \mathrm{sr}^{-1}\right)$. The values of $G_{0}$ between the star and the PDR were obtained assuming geometrical dilution of the stellar radiation field and a local extinction value of $A_{\mathrm{V}}=1.5$. Inside the PDR, we used the fraction of carbon locked in eVSG as a probe for $G_{0}$ (Pilleri et al. 2012).

the peak of the $\mathrm{H}_{2} \mathrm{~S}(3)$ line, which is found at a magnitude of visual extinction, $A_{\mathrm{V}} \sim 1$ (Pilleri et al. 2012).

Figures 5a,b show the integrated intensity maps of the $3.3 \mu \mathrm{m}$ and $3.4 \mu \mathrm{m}$ bands. Both maps peak toward the $\mathrm{H}_{2} \mathrm{~S}(3)$ filament that delineates the PDR front. The $3.3 \mu \mathrm{m}$ band is also detected in the cavity between the star and the PDR, whereas the $3.4 \mu \mathrm{m}$ band is detected only toward the PDR. Neither the $3.3 \mu \mathrm{m}$ nor the $3.4 \mu \mathrm{m}$ bands is detected in the more shielded layers of the PDR, farther away from HD 200775 than the $\mathrm{H}_{2}$ filaments.

In Figs. 5c, d we compare these maps with the spatial distribution of $\mathrm{PAH}^{0}$ and eVSGs intensities obtained with PAHTAT. A very good spatial correlation is found between $I_{3.3}$ and $I_{\mathrm{PAH}^{0}}$ (Fig. 5c). In contrast, the $3.4 \mu \mathrm{m}$ band is more localized on the 


\section{P. Pilleri et al.: The mixed aliphatic/aromatic composition of eVSGs in NGC 7023 revealed by the $3.4 / 3.3 \mu \mathrm{m}$ ratio}
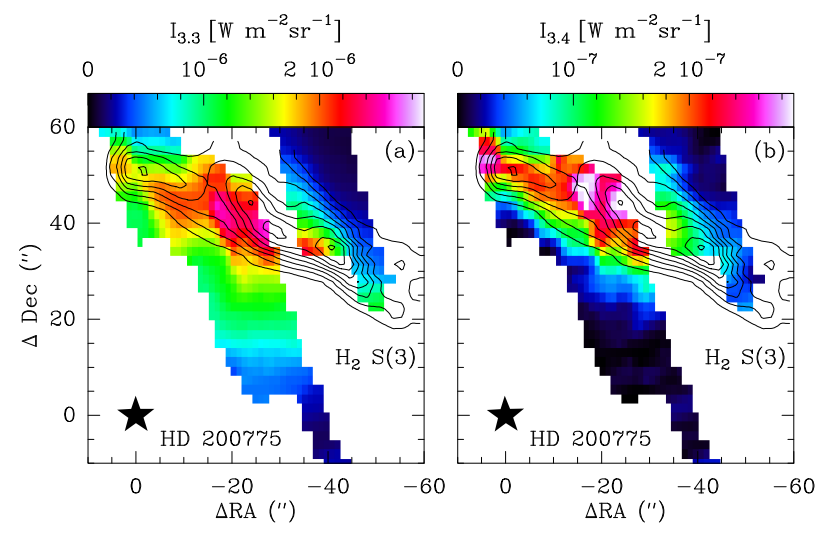

to the PDR front and mask the pixels that have $I_{\mathrm{PAH}^{+}} / I_{\mathrm{PAH}^{0}}>1$ and those where the $3.4 \mu \mathrm{m}$ band is not detected.

\section{Discussion}

\subsection{Variation of $I_{3.3}$ with $G_{0}$}

The dependence of the $3.3 \mu \mathrm{m}$ band intensity on $G_{0}$ results from three effects: change in the abundance of its carriers, PAH ionization since the $3.3 \mu \mathrm{m}$ band is expected to be smaller for $\mathrm{PAH}^{+}$ relative to $\mathrm{PAH}^{0}$ (see a review on quantum-chemical calculations by Pauzat 2011), and excitation effects. We discuss these effects here by considering the $I_{3.3} / I_{\mathrm{PAH}^{0}}$ ratio. As shown in Fig. 6a, this ratio presents some dispersion, but with an overall increase with $G_{0}$.

The observed intensity of the $3.3 \mu \mathrm{m}$ band can be written as a function of the column density $N_{3.3}$ of its carriers and $G_{0}$ :

$I_{3.3}=G_{0} N_{3.3} \epsilon_{3.3}$,

where $\epsilon_{3.3}$ is the average emissivity at $3.3 \mu \mathrm{m}$ per aromatic $\mathrm{CH}$ bond in PAHs along the line of sight, which depends on the molecular properties of their carriers and the excitation conditions, and $N_{3.3}$ is the column density of aromatic $\mathrm{CH}$ bonds.

The intensity of the $3.3 \mu \mathrm{m}$ band is the sum of the contribution of the neutral and cationic PAHs. Thus, the ratio $I_{3.3} / I_{\mathrm{PAH}^{0}}$ can be written as

$\frac{I_{3.3}}{I_{\mathrm{PAH}^{0}}}=\frac{I_{3.3}^{0}}{I_{\mathrm{PAH}^{0}}}\left(1+\frac{I_{3.3}^{+}}{I_{3.3}^{0}}\right)=\frac{N_{3.3}^{0}}{N_{\mathrm{PAH}^{0}}} \frac{\epsilon_{3.3}^{0}}{\epsilon_{\mathrm{PAH}}^{0}}\left(1+\frac{N_{3.3}^{+}}{N_{3.3}^{0}} \frac{\epsilon_{3.3}^{+}}{\epsilon_{3.3}^{0}}\right)$,
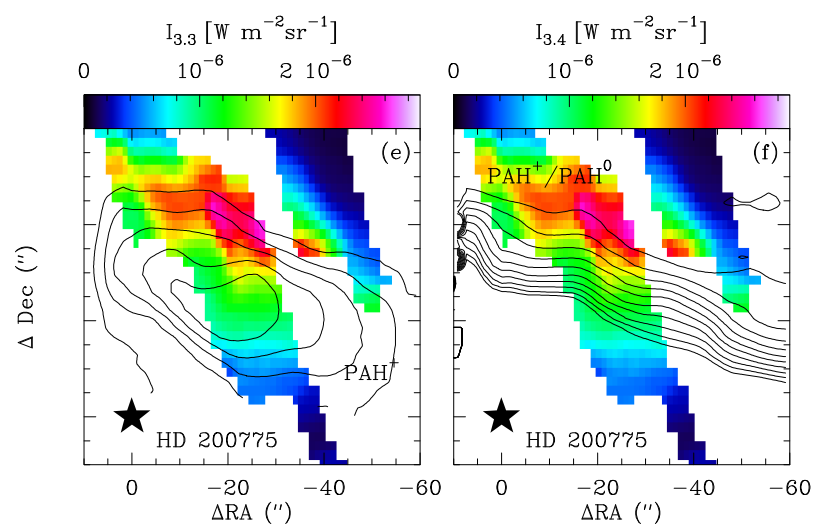

where $I_{3.3}^{0 /+}, \epsilon_{3.3}^{0 /+}$ are the emitted intensity and average emissivity of the $3.3 \mu \mathrm{m}$ band due to $\mathrm{PAH}^{0}$ and $\mathrm{PAH}^{+}$, and $N_{3.3}^{0 /+}$ is the column density of aromatic $\mathrm{CH}$ bonds of neutral and ionized species. We assume that the ionization fraction is the same for the carriers of the $3.3 \mu \mathrm{m}$ band and for the whole PAH population, that is, $N_{3.3}^{+} / N_{3.3}^{0}=N_{\mathrm{PAH}^{+}} / N_{\mathrm{PAH}^{0}}$ (Montillaud et al. 2013, see discussion below). From this we obtain

$\frac{I_{3.3}}{I_{\mathrm{PAH}^{0}}}=k_{3.3}\left(1+\frac{N_{\mathrm{PAH}^{+}}}{N_{\mathrm{PAH}^{0}}} p_{3.3}\right)$

where we have defined

$k_{3.3}=\frac{\epsilon_{3.3}^{0}}{\epsilon_{\mathrm{PAH}}^{0}} \frac{N_{3.3}^{0}}{N_{\mathrm{PAH}^{0}}}$

grated intensity as observed by the IRC instrument (color scale). The contours represent the integrated intensity of the $\mathrm{H}_{2} \mathrm{~S}(3)$ line at $9.7 \mu \mathrm{m}$ (linear steps of $1.7 \times 10^{-7} \mathrm{~W} \mathrm{~m}^{-2} \mathrm{sr}^{-1}$ ), and the star indicates the position of HD 200775. Middle: comparison of the integrated intensity of the bands at 3.3 and $3.4 \mu \mathrm{m}$ (color scale) with the spatial distribution of the $\mathrm{PAH}^{0}$ and eVSG intensity, respectively (contours steps of $1.7 \times 10^{-5} \mathrm{~W} \mathrm{~m}^{2} \mathrm{sr}^{-1}$, starting at $\left.1.7 \times 10^{-5} \mathrm{~W} \mathrm{~m}^{2} \mathrm{sr}^{-1}\right)$. Bottom: comparison of the integrated intensity of the $3.3 \mu \mathrm{m}$ band with that of $\mathrm{PAH}^{+}$ and with the ratio $I_{\mathrm{PAH}^{+}} / I_{\mathrm{PAH}^{0}}$. In f) the contours are shown in steps of 0.3 , with increasing values while approaching the star from the PDR.

dense PDR with a good spatial correlation between $I_{3.4}$ and $I_{\mathrm{eVSG}}$ (Fig. 5d). Still, the $3.3 \mu \mathrm{m}$ band is observed inside the cavity, which indicates that there is a contribution from $\mathrm{PAH}^{+}$to its emission, and/or that a fraction of $\mathrm{PAH}^{0}$ is present in the cavity (Fig. 5e). The intensity at $3.3 \mu \mathrm{m}$ decreases by a factor $>5$ (from $3 \times 10^{-6}$ to $\left.6 \times 10^{-7} \mathrm{~W} \mathrm{~m}^{-2} \mathrm{sr}^{-1}\right)$ in regions where $I_{\mathrm{PAH}^{+}} / I_{\mathrm{PAH}^{0}} \gtrsim 3$ (Fig. 5f). Since we are interested in the evolution of the $3.4 \mu \mathrm{m}$ band relative to the $3.3 \mu \mathrm{m}$ band, we restrain our analysis below

$k_{3.3}$ represents the fraction of the bolometric PAH emission in the $3.3 \mu \mathrm{m}$ band for the $\mathrm{PAH}^{0}$ population and is therefore an indicator of the PAH size distribution. Pech et al. (2002) have modeled the IR emission spectrum of a distribution of PAHs under irradiation by UV photons and derived values for $k_{3.3}$ of about 0.01-0.02 depending on the assumed size distribution. The $p_{3.3}$ parameter represents the emissivity ratio for PAH cations and neutrals. It is expected to depend on the excitation conditions since PAH cations (open-shell species) have more electronic bands at low energy. However, in a relatively hard UV field, the excitation will be dominated by the strong UV $\pi \rightarrow \pi^{*}$ and $\sigma \rightarrow \sigma^{*}$ type bands, which are present in all charge states and are almost equal across them (Mulas et al. 2006). The $p_{3.3}$ parameter will then depend mainly on the relative IR absorption crosssection at $3.3 \mu \mathrm{m}$ of PAH cations relative to neutrals. Values in 

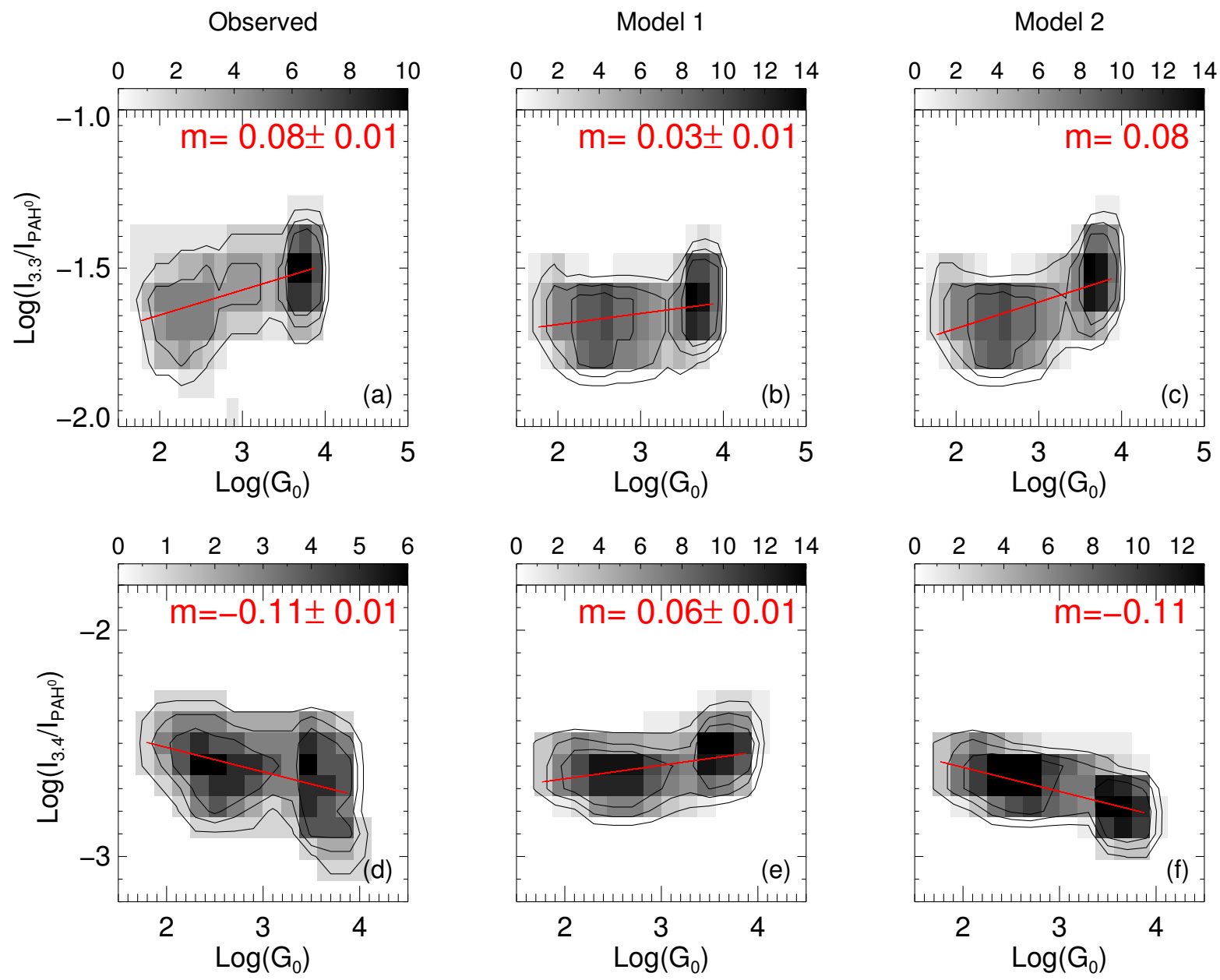

Fig. 6. First column: correlation between the observed $I_{3.3} / I_{\mathrm{PAH} 0}$ and $I_{3.4} / I_{\mathrm{PAH} 0}$ as a function of $G_{0}$. Second column: same plots for model maps of $I_{3.3} / I_{\mathrm{PAH} 0}$ and $I_{3.4} / I_{\mathrm{PAH} 0}$ computed using Eqs. (6) and (8) and the obtained values for $I_{\mathrm{PAH}^{+}} / I_{\mathrm{PAH}^{0}}$ (see text for details). Third column: semi-empirical model that in addition includes the dependency with $G_{0}$ on the intensity at 3.3 and $3.4 \mu \mathrm{m}$ relative to all $\mathrm{PAH}^{0}$ bands (see text). In all panels, the linear fit to the data points (red line) is shown as well as its slope $m$.

the range $0.2-0.5$ have been calculated for PAH sizes between 20 and $60 \mathrm{C}$ atoms (Malloci et al. 2007).

The total emissivity for a neutral and a cation with similar size is approximately the same because of conservation of energy and the fact that their UV absorption cross-sections are in first approximation similar, as explained above. Therefore, we can use the ratio of the observed intensity $I_{\mathrm{PAH}^{+}} / I_{\mathrm{PAH}^{0}}$ as a probe for the column density ratio of ionized to neutral PAHs, and rewrite Eq. (3) using only the observed intensities and the molecular parameters $k_{3.3}$ and $p_{3.3}$ :

$\frac{I_{3.3}}{I_{\mathrm{PAH}^{0}}}=k_{3.3}\left(1+\frac{I_{\mathrm{PAH}^{+}}}{I_{\mathrm{PAH}^{0}}} p_{3.3}\right)$.

The term in parenthesis is related to the contribution of the ionization fraction of PAHs along the line of sight to the observed $I_{3.3} / I_{\mathrm{PAH}^{0}}$ ratio. Using the maps we obtained with PAHTAT, we derived the values of $G_{0}$ and $I_{\mathrm{PAH}^{+}} / I_{\mathrm{PAH}^{0}}$ for each pixel (Figs. 4 and $5 \mathrm{f}$, respectively). Then, we used these values to compute a model map of $I_{3.3} / I_{\mathrm{PAH}^{0}}$ using Eq. (6) with constant values of $k_{3.3}$ and $p_{3.3}$. This semi-empirical model (hereafter model 1, Fig. 6b) cannot reproduce the observed trend in $I_{3.3} / I_{\mathrm{PAH}^{0}}$. As an example, Fig. 6 b shows the results for model 1 using $k_{3.3}=0.2$ and $p_{3.3}=0.5$. These values give the best fit to the data within the physically meaningful range for $k_{3.3}$ and $p_{3.3}$. This shows that the observed variations of $I_{3.3} / I_{\mathrm{PAH}^{0}}$ cannot be accounted for by a variation in the mean charge of PAHs alone.
To obtain a better fit of the observed increase of $I_{3.3} / I_{\mathrm{PAH}}{ }$ with $G_{0}$, we assumed that $k_{3,3}$ depends on $G_{0}$ with a linear relationship: $k_{3.3}=0.02+k_{3.3}^{*} \times G_{0}$. We fit the slope of this correlation by varying $k_{3,3}^{*}$. The best fit is obtained using $k_{3,3}^{*}=8 \times 10^{-7}$ (hereafter model 2 , Fig. $6 \mathrm{c}$ ). The dependency of $k_{3.3}$ on $G_{0}$ could be due to an increase of the abundance of the $3.3 \mu \mathrm{m}$ carrier relative to all neutral PAHs, or to an increase of the emissivity at $3.3 \mu \mathrm{m}$ relative to the total emissivity of $\mathrm{PAH}^{0}$. As discussed below, the first effect is likely to involve a chemical evolution of the PAH population, whereas the second is related to excitation conditions.

Analyzing infrared observations obtained by Spitzer and Herschel, Berné \& Tielens (2012) showed that there is no global variation of the PAH abundance in the region covered by our observations. Montillaud et al. (2013) modeled the chemical evolution of PAHs in NGC 7023 NW. They showed that PAHs of small sizes (with a number of carbon atoms $N_{\mathrm{C}} \lesssim 50$ ) are fully dehydrogenated, whereas larger PAHs are normally hydrogenated and possibly superhydrogenated for the largest sizes $\left(N_{\mathrm{C}} \gtrsim 90\right)$. The ionization fraction is found to be rather similar from one size to the other. It is interesting to note that across the PDR, intermediate-sized PAHs $\left(N_{\mathrm{C}} \sim 60-70\right)$ can experience strong variations in their hydrogenation state because of the competition between photodissociation by UV photons and recombination with $\mathrm{H}$ atoms. For instance, $\mathrm{C}_{66} \mathrm{H}_{20}$ is predicted to be fully 
hydrogenated at the border of the PDR, but fully dehydrogenated deeper inside the cloud at a distance from 5 to $10^{\prime \prime}$ from the PDR front. Here, the abundance of $\mathrm{H}$ relative to $\mathrm{H}_{2}$ decreases faster than the attenuation of UV photons, resulting in fully dehydrogenated species. This effect could explain the increase in the $3.3 \mu \mathrm{m}$ band intensity with increasing $G_{0}$. Another possibility is that the emissivity at $3.3 \mu \mathrm{m}$ increases with $G_{0}$ as a result of the higher average internal temperatures of PAHs with higher UV field. Two effects may be invoked: in the most exposed layers of the PDR, the UV radiation field is becoming harder because of decreased extinction by dust (Rapacioli et al. 2006). Secondly, when the UV flux increases, the probability for multiple photon events increases. A PAH that has absorbed a UV photon can only relax part of its internal energy before absorbing another photon, and it is therefore heated to higher temperatures. However, whereas multiple photon events are important for studying photo-dissociation (Montillaud et al. 2013), they remain rare events in NGC $7023 \mathrm{NW}$ and do not contribute significantly to the mid-IR emission. In addition, Witt et al. (2006) showed that in the first layers of the PDR there is a significant contribution of high-energy UV-photons $(h v \gtrsim 10.3 \mathrm{eV})$ that are suppressed in the more protected layers $\left(A_{\mathrm{V}} \gtrsim 2\right)$. The variation of the hardness of the UV field across the PDR provides another explanation for the increase of the intensity of the $3.3 \mu \mathrm{m}$ band with $G_{0}$. Quantifying the importance of this process compared to the hydrogenation effect described above would require a full chemical and photo-physical model, which is not the purpose of this paper. We conclude that the increase of $I_{3.3} / I_{\mathrm{PAH}^{0}}$ with $G_{0}$ is due to a change in the hardness of the UV field combined with an increased abundance of aromatic $\mathrm{CH}$ bonds at the border of the PDR, both effects being relatively moderate.

\subsection{Photo-destruction of aliphatics}

Similar to the $3.3 \mu \mathrm{m}$ band, the intensity of the $3.4 \mu \mathrm{m}$ band relative to the total intensity of the $\mathrm{PAH}^{0}$ bands $\left(I_{3.4} / I_{\mathrm{PAH}^{0}}\right)$ also presents some dispersion. However, it shows an overall decrease with $G_{0}$ (Fig. 6d). In analogy with the $3.3 \mu \mathrm{m}$ case, the observed intensity of the $3.4 \mu \mathrm{m}$ band can be written as

$I_{3.4}=G_{0} N_{3.4} \epsilon_{3.4}$,

where $\epsilon_{3.4}$ and $N_{3.4}$ are the average emissivity and column density per aliphatic $\mathrm{CH}$ bond along the line of sight. Equation (6) can be rewritten for the $3.4 \mu \mathrm{m}$ band as

$\frac{I_{3.4}}{I_{\mathrm{PAH}^{0}}}=k_{3.4} \times\left(1+\frac{I_{\mathrm{PAH}^{+}}}{I_{\mathrm{PAH}^{0}}} \times p_{3.4}\right)$,

where we have defined

$k_{3.4}=\frac{\epsilon_{3.4}^{0}}{\epsilon_{\mathrm{PAH}}^{0}} \frac{N_{3.4}^{0}}{N_{\mathrm{PAH}^{0}}}=\frac{I_{3.4}^{0}}{I_{\mathrm{PAH}}^{0}}$

$p_{3.4}=\frac{\epsilon_{3.4}^{+}}{\epsilon_{3.4}^{0}}$

To obtain an estimate of the typical values for $p_{3.4}$, we used the NASA-Ames PAH IR Spectroscopic database ${ }^{3}$ (hereafter the NASA-Ames PAH database, Boersma et al. 2014) and obtained $p_{3.4} \gtrsim 1$, depending on the size of the PAH being considered. Since $p_{3.4}>p_{3.3}$, the term in parenthesis is larger for the $3.4 \mu \mathrm{m}$ than for the $3.3 \mu \mathrm{m}$ band, implying that the charge state of PAHs modifies the ratio $I_{3.4} / I_{\mathrm{PAH}^{0}}$ more significantly than does $I_{3.3} / I_{\mathrm{PAH}^{0}}$.

3 http://www.astrochem.org/pahdb/
Assuming $p_{3.4}=1$, the best fit to the data using our model 1 is obtained using $k_{3.4}=0.002$, which allows us to reproduce the average values of $I_{3.4} / I_{\mathrm{PAH}^{0}}$ (Fig. 6e). $k_{3.4}$ is found to be a factor of 10 lower than $k_{3.3}$ because of the lower abundance of aliphatic $\mathrm{CH}$ groups compared to the aromatic $\mathrm{CH}$ groups. The relative abundance of aliphatic vs. aromatic groups we derive agrees with previous studies, including the recent work of $\mathrm{Li}$ \& Draine (2012). The observed negative slope of the $I_{3.4} / I_{\mathrm{PAH}^{0}}$ vs. $G_{0}$ correlation (Fig. $6 \mathrm{~d}$ ) is not reproduced by our model 1 (Eq. (8) and Fig. 6e). As for the $3.3 \mu \mathrm{m}$ band, we assume a dependency of $k_{3.4}$ with $G_{0}: k_{3.4}=0.002+k_{3.4}^{*} \times G_{0}$. The observations can be best reproduced by $k_{34}^{*}=-1.8 \times 10^{-7}$ (Fig. 6e). This negative term mainly reflects the destruction of the carriers of the $3.4 \mu \mathrm{m}$ band with increasing $G_{0}$. This is an effective value that reflects both the increase in intensity and hardness of the UV field while reaching the edge of the PDR.

\subsection{Variation of $I_{3.4} / I_{3.3}$ with $G_{0}$}

The ratio $I_{3.4} / I_{3.3}$ can be used as a tracer of the aliphatic to aromatic content in PAHs if we assume that both bands arise from the same carriers. This ratio is low in the cavity $(\sim 0.03$ at P1) and increases monotonically toward the PDR, reaching its highest value $(\sim 0.15)$ slightly behind the $\mathrm{H}_{2} \mathrm{~S}(3)$ and the $\mathrm{PAH}^{0}$ emission (Fig. 7a). The highest gradient is found at the PDR front where in only $\sim 10^{\prime \prime}$ the ratio increases by a factor of $\sim 3$. This shows that photo-chemical processing of the carriers of the $3.4 \mu \mathrm{m}$ band is taking place in this region. We note that $10^{\prime \prime}$ corresponds to the angular width of the filament traced by the $\mathrm{H}_{2}$ rotational emission. Interestingly, this is also the evaporation zone of eVSGs into gas-phase PAHs. We have used the $I_{\mathrm{eVSG}} / I_{\mathrm{PAH}}=I_{\mathrm{eVSG}} /\left(I_{\mathrm{PAH}^{0}}+I_{\mathrm{PAH}^{+}}\right)$ratio as an indicator of the destruction of eVSGs into PAHs by UV photons. The relative spatial distributions suggest that the destruction of eVSGs is then followed by that of the $3.4 \mu \mathrm{m}$ carriers. This suggests that the evaporation of eVSGs leads to the production of PAHs with aliphatic groups.

Figure $7 \mathrm{c}$ shows the pixel-to-pixel correlation between $I_{3.4} / I_{3.3}$ and $\log \left(G_{0}\right)$. Dividing each side of Eq. (8) by Eq. (6), we obtain

$\frac{I_{3.4}}{I_{3.3}}=\frac{N_{3.4}}{N_{3.3}} \frac{\epsilon_{3.4}^{0}}{\epsilon_{3.3}^{0}} D_{\text {ion }}$

where $D_{\text {ion }}$ is the ratio of the terms in parenthesis in each equation and represents the variation of $I_{3.4} / I_{3.3}$ due to ionization. Because the intensity of $\mathrm{PAH}^{+}$relative to that of $\mathrm{PAH}^{0}$ is $\lesssim 0.4$, $D_{\text {ion }}$ is very close to 1 . More specifically, $D_{\text {ion }}$ is about $10 \%$ higher in the more exposed regions in which $I_{\mathrm{PAH}^{+}} / I_{\mathrm{PAH}^{0}}=0.4$ compared to the region with no $\mathrm{PAH}^{+}$emission, thus it cannot explain the trend shown in Fig. 7c. For PAH sizes larger than $\sim 50$ carbon atoms, $\epsilon_{3.4}^{0} / \epsilon_{3.3}^{0}$ can be also considered constant (cf. NASA-Ames PAH database), and therefore the observed trend can be mainly attributed to a decrease in the abundance of the aliphatic $\mathrm{CH}$ bonds relative to aromatic $\mathrm{CH}$ bonds.

The trend we observe is similar to that reported in Joblin et al. (1996) and analyzed by the authors using a simple photochemical model that describes the evolution of methylated PAHs. The authors showed that the decrease of the $3.4 \mu \mathrm{m}$ band occurs because the reactions that could reconstruct the aliphatic side-groups in the gas-phase are highly improbable. 

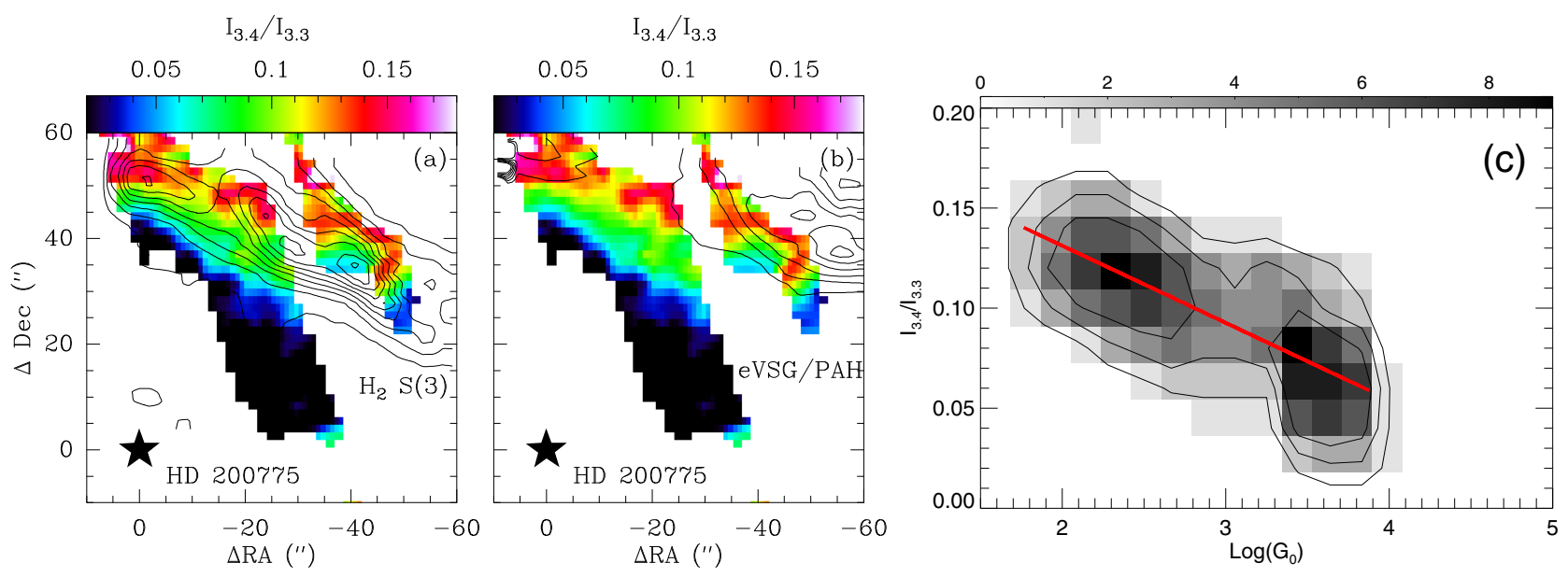

Fig. 7. Map of the $3.4 / 3.3 \mu \mathrm{m}$ ratio (color scale) with the contours of the $\mathrm{H}_{2} \mathrm{~S}(3)$ line a), and of the ratio $I_{\mathrm{eVSG}} / I_{\mathrm{PAH}}=I_{\mathrm{eVSG}} /\left(I_{\mathrm{PAH}}{ }+I_{\mathrm{PAH}}+\right)$ b) The density plot of the $I_{3.4} / I_{3.3}$ band ratios vs. $G_{0}$ is also shown c). In red, we report the linear regression of the data points. Only the pixels for which $I_{3.3}>1.0 \times 10^{-7} \mathrm{~W} \mathrm{~m}^{-2} \mathrm{sr}^{-1}$ have been used to build in the analysis of the $3.4 / 3.3 \mu \mathrm{m}$ ratio.

\section{Conclusions}

Thanks to the high spatial resolution of observations made in the near- and mid-IR, we probed the evolution of aliphatic and aromatic $\mathrm{CH}$ groups in the spatially resolved PDR NGC 7023 NW. We showed that the intensity of the $3.3 \mu \mathrm{m}$ band relative to the total PAH emission increases with $G_{0}$, while the relative contribution of the $3.4 \mu \mathrm{m}$ band decreases with $G_{0}$. In the most exposed layers of the PDR, the UV radiation field is harder and high-energy UV photons can excite PAHs to higher temperatures, leading thus to an increase of the fraction of energy emitted at $3.3 \mu \mathrm{m}$. The higher abundance of $\mathrm{H}$ atoms in this region can also increase the abundance of $\mathrm{CH}$ aromatic bonds. On the other hand, the higher flux of UV photons leads to efficient destruction of the more fragile aliphatic $\mathrm{CH}$ bonds attached to PAHs, which cannot be efficiently reformed in these regions. In addition, we showed that the observed $3.45 \mu \mathrm{m}$ plateau is dominated by the emission from aromatic bonds.

We find that the change in the aliphatic vs. aromatic composition is particularly important along the filament that delineates the PDR. In particular, the $I_{3.4} / I_{3.3}$ ratio peaks close to the region where eVSGs are photo-evaporated into PAHs. This suggests that the processing of eVSGs leads to PAHs with attached aliphatic sidegroups, which provides further insights into the formation and evolution of these species in astrophysical environments. (Very) small grains of mixed aromatic and aliphatic composition are known to be present in circumstellar environments (Kwok et al. 2001; Goto et al. 2003; Sloan et al. 2007), and they have been considered to be a major component in the dust model by Jones (2012). We provided here the first observational insights for the presence of such grains in interstellar clouds.

Acknowledgements. We thank the anonymous referee for useful comments. This work was supported by the French National Program, Physique et Chimie $\mathrm{du}$ Milieu Interstellaire, which is gratefully acknowledged. P. Pilleri acknowledges financial support from the Centre National d'Études Spatiales (CNES). The research leading to these results has also received funding from the European Research Council under the European Union's Seventh Framework Programme (FP/2007-2013) ERC-2013-SyG, Grant Agreement No. 610256 NANOCOSMOS.

\section{References}

Alecian, E., Catala, C., Wade, G. A., et al. 2008, MNRAS, 385, 391 Allamandola, L. J., Tielens, A. G. G. M., \& Barker, J. R. 1985, ApJ, 290, L25 Allamandola, L. J., Tielens, A. G. G. M., \& Barker, J. R. 1989, ApJS, 71, 733
Barker, J. R., Allamandola, L. J., \& Tielens, A. G. G. M. 1987, ApJ, 315, L61

Benisty, M., Perraut, K., Mourard, D., et al. 2013, A\&A, 555, A113

Berné, O., \& Tielens, A. G. G. M. 2012, Proc. Nat. Acad. Sci., 109, 401

Berné, O., Joblin, C., Deville, Y., et al. 2007, A\&A, 469, 575

Bernstein, M. P., Sandford, S. A., \& Allamandola, L. J. 1996, ApJ, 472, L127

Boersma, C., Bauschlicher, Jr., C. W., Ricca, A., et al. 2014, ApJS, 211, 8

Dartois, E., Muñoz Caro, G. M., Deboffle, D., \& d'Hendecourt, L. 2004, A\&A, 423, L33

Finkenzeller, U. 1985, A\&A, 151, 340

Fuente, A., Martin-Pintado, J., Cernicharo, J., \& Bachiller, R. 1993, A\&A, 276, 473

Fuente, A., Martin-Pintado, J., Neri, R., Rogers, C., \& Moriarty-Schieven, G. 1996, A\&A, 310, 286

Geballe, T. R., Tielens, A. G. G. M., Allamandola, L. J., Moorhouse, A., \& Brand, P. W. J. L. 1989, ApJ, 341, 278

Gerin, M., Phillips, T. G., Keene, J., Betz, A. L., \& Boreiko, R. T. 1998, ApJ, 500,329

Goto, M., Gaessler, W., Hayano, Y., et al. 2003, ApJ, 589, 419

Habing, H. J. 1968, Bull. Astron. Inst. Netherlands, 19, 421

Joblin, C., Leger, A., \& Martin, P. 1992, ApJ, 393, L79

Joblin, C., Tielens, A. G. G. M., Allamandola, L. J., \& Geballe, T. R. 1996, ApJ, 458,610

Jones, A. P. 2012, A\&A, 540, A1

Jourdain de Muizon, M., D’Hendecourt, L. B., \& Geballe, T. R. 1990, A\&A, 235, 367

Kwok, S., Volk, K., \& Bernath, P. 2001, ApJ, 554, L87

Léger, A., \& Puget, J. L. 1984, A\&A, 137, L5

Lemaire, J. L., Field, D., Gerin, M., et al. 1996, A\&A, 308, 895

Li, A., \& Draine, B. T. 2012, ApJ, 760, L35

Malloci, G., Joblin, C., \& Mulas, G. 2007, Chem. Phys., 332, 353

Martini, P., Sellgren, K., \& Hora, J. L. 1997, ApJ, 484, 296

Montillaud, J., \& Joblin, C. 2014, A\&A, 567, A45

Montillaud, J., Joblin, C., \& Toublanc, D. 2013, A\&A, 552, A15

Mori, T. I., Onaka, T., Sakon, I., et al. 2014, ApJ, 784, 53

Mulas, G., Malloci, G., Joblin, C., \& Toublanc, D. 2006, A\&A, 460, 93

Murakami, H., Baba, H., Barthel, P., et al. 2007, PASJ, 59, 369

Onaka, T., Matsuhara, H., Wada, T., et al. 2007, PASJ, 59, 401

Pauzat, F. 2011, in EAS Pub. Ser. 46, eds. C. Joblin, \& A. G. G. M. Tielens, 75

Pech, C., Joblin, C., \& Boissel, P. 2002, A\&A, 388, 639

Peeters, E., Hony, S., Van Kerckhoven, C., et al. 2002, A\&A, 390, 1089

Pilleri, P., Montillaud, J., Berné, O., \& Joblin, C. 2012, A\&A, 542, A69

Rapacioli, M., Joblin, C., \& Boissel, P. 2005, A\&A, 429, 193

Rapacioli, M., Calvo, F., Joblin, C., et al. 2006, A\&A, 460, 519

Simon, A., \& Joblin, C. 2009, J. Phys. Chem. A, 113, 4878

Sloan, G. C., Bregman, J. D., Geballe, T. R., Allamandola, L. J., \& Woodward,

C. E. 1997, ApJ, 474, 735

Sloan, G. C., Jura, M., Duley, W. W., et al. 2007, ApJ, 664, 1144

Steglich, M., Jäger, C., Huisken, F., et al. 2013, ApJS, 208, 26

Tielens, A. G. G. M. 2005, The Physics and Chemistry of the Interstellar Medium (Cambridge, UK: Cambridge University Press)

Weingartner, J. C., \& Draine, B. T. 2001, ApJ, 548, 296

Werner, M. W., Uchida, K. I., Sellgren, K., et al. 2004, ApJS, 154, 309

Witt, A. N., Gordon, K. D., Vijh, U. P., et al. 2006, ApJ, 636, 303 